munikation (je als Quelle »LFI« für Landesförderinstitut). Zudem fließen Erkenntnisse aus den Fallstudien-Interviews mit der hessischen WIBank und der sachsen-anhaltischen Investitionsbank sowie mit der KfW-Bank und dem Bundesinnenministerium in die Ausarbeitung ein (vgl. Kapitel 6.2.2). Die in Kapitel 6.2.3 aufbereiteten Inhalte der Landesförderprogramme zum barrierefreien bzw. -reduzierten Wohnraum basieren auf der Auswertung einer umfangreichen Matrix, die auf Nachfrage bei der Autorin einsehbar ist.

Datenbasis und Strukturierung bei den Wohnberatungsstellen

Die empirische Untersuchung der politischen Bedeutung der Wohnberatungsstellen zur Wohnungsanpassung stellt eine methodische Herausforderung dar, da ihre politische Steuerung weder in Gesetzestexten noch in Richtlinien strukturiert nachzuvollziehen sind. Teils handelt es sich auch um eine wirtschaftliche Selbstregelung, da die Beratungen vollends über Träger der freien Wohlfahrtspflege, Architektenkammern oder Wohnungsunternehmen übernommen werden. Viele Bundesländer fördern derartige Beratungsstrukturen aber in finanzieller oder organisatorischer Form, sodass durchaus von einer politischen Steuerung zu sprechen ist. Um sich dieser undurchsichtigen Struktur zu nähern, kann auf einige Studien zu Wohnberatungsstellen zurückgegriffen werden (BMVBS 2011; BMVBS 2013; Schneider 2013; KDA/Wüstenrot Stiftung 2014a; Lehrmacher-Dubberke et al. 2015; Joo 2018).

Somit basiert diese Analyse zunächst auf der vorhandenen Studienlage, welche aufbereitet und über Expert*inneninterviews mit Wohnberater*innen validiert, aktualisiert und auf den eigenen Schwerpunkt der politischen Prozesse hin konkretisiert wird. Über die Bundesarbeitsgemeinschaft Wohnungsanpassung e.V. lassen sich regionale Ansprechpartner*innen mit einem Überblick über die Strukturen im eigenen Bundesland identifizieren. Durch schriftliche Anfragen kommen telefonische, persönliche und teils vertiefende schriftliche Kontakte mit insgesamt sechs Bundesländern zustande (die Gespräche finden zwischen Oktober 2019 und Februar 2020 statt). Ferner sind auch hier Informationen aus den beiden Fallstudien zu nutzen, in dessen Rahmen ohnehin mit sachsen-anhaltischen und hessischen Wohnberater*innen gesprochen wird (vgl. Kapitel 5.2.2). Die Auswertung erfolgt entlang des erstellten Leitfadens in der Kapitelstruktur der Kapitel 6.3.1 bis 6.3.3.

Zusammengefasst orientiert sich die Datenbasis aus vorhandenen Sekundärdaten und erhobenen Primärdaten an der empirischen Verfügbarkeit. Zur Auswertung wird für jede einzelne Policy zum barrierefreien bzw. -reduzierten Wohnraum geprüft, welche Kategorien zielführend zur Beschreibung der Maßnahme und zur Beurteilung der Varianz zwischen den 16 Ländern sind.

\title{
5.2 Politikfeldanalytische Fallstudien
}

Aufbauend auf der Typologie der politischen Maßnahmen werden politikfeldanalytische Fallstudien für die Bundesländer Sachsen-Anhalt und Hessen durchgeführt (vgl. Kapitel 7). Das Ziel ist es, die hinter den Landes-Policies zum barrierefreien bzw. -reduzierten Wohnraum liegenden politischen Prozesse zu verstehen und zu erklären. Mit 
Blatter et al. (2018: 168) lässt sich das hier gewählte qualitative Vorgehen in einem Feld mit wenig wissenschaftlicher Expertise wie folgt erklären: »Typischerweise stehen am Beginn von Forschungsprogrammen Einzelfallstudien, dann werden vergleichende Fallstudien und schließlich statistische Analysen mit vielen Fällen durchgeführt « (vgl. Forschungsstand in Kapitel 3.3). Nach Jahn (2013: 247f.) können Fallstudien außerdem nicht nur detaillierte Erkenntnisse über die betreffenden Phänomene generieren, sondern ermöglichen es zudem, aus den Informationen zu einem bestimmten Fall Erklärungsfaktoren zu identifizieren, die womöglich übergeordnete Gültigkeit besitzen (vgl. auch Blatter et al. 2007: 123ff.; Egner 2019a: 46ff.). Aufgrund der Jungfräulichkeit der politikfeldanalytischen Forschung zur Barrierefreiheit bzw. -reduzierung erscheinen qualitative Fallstudien folglich als eine geeignete Methode.

Dafür ist der in Kapitel 4 ausgearbeitete Ansatz eigendynamischer politischer Prozesse von Böcher/Töller (2012) der zentrale Ausgangspunkt. Die darin deduktiv hergeleiteten Annahmen sind im Verlauf der Forschung entweder um weitere, nachträglich als wichtig aufscheinende Aspekte zu ergänzen oder umgekehrt zu verschlanken, sollten sich vorab entwickelte Annahmen als unzutreffend herausstellen. Damit wird dem Umstand Rechnung getragen, dass qualitative Forschung oft sowohl hypothesentestend als auch hypothesengenerierend vorgehen muss (Muno 2015: 81; Reiter/Töller 2014: 30; Jahn 2013: 164f.).

Generell geht die Politikfeldanalyse empirisch-analytisch vor: Der Fokus liegt nicht auf normativen »Es-sollte«-Aussagen, sondern auf erklärenden »Es-ist-so,-weil«Erkenntnissen (vgl. Jahn 2013: 194). Es geht um das Verstehen und Erklären der politischen Prozesse im wohnungspolitischen Teilbereich des barrierefreien bzw. -reduzierten Wohnraums. Dafür wird im Folgenden die methodische Grundlage dargestellt, beginnend mit der Auswahl der Fallstudienländer Sachsen-Anhalt und Hessen (Kapitel 5.2.1), gefolgt von den genutzten Sekundärdaten sowie der eigenen Datenerhebung über leitfadengestützte Interviews (Kapitel 5.2.2) bis hin zur Aufbereitung und Auswertung des Datenmaterials in Form einer qualitativen Inhaltsanalyse (Kapitel 5.2.3).

\subsubsection{Auswahl der Fallstudien}

Das methodische Design von Fallstudien ist untrennbar mit einer Begründung der ausgewählten Fälle verbunden. Auch wenn die vorliegende Arbeit keinen strengen Fallvergleich, sondern die »Identifikation von kausalen Mechanismen durch Prozessanalysen « anstrebt (Blatter et al. 2018: 170ff.), ist eine bewusste Fallauswahl aus den 16 Bundesländern unabdingbar. Diese kann prinzipiell entlang von räumlichen oder analytischen Kriterien erfolgen, genauso aber als positive oder negative Fallauswahl. ${ }^{2}$

Der für die vergleichende Politikwissenschaft paradigmatische Leitsatz zur maximierten experimentellen Varianz, minimierten Fehlervarianz und kontrollierten externen Varianz (vgl. Pickel 2016: 28) kann für die vorliegende Forschungsarbeit jedoch nur

Die Entscheidung für zwei Einzelfallstudien darf nicht mit einem Paarvergleich verwechselt werden (vgl. Jahn 2013: 226ff., 248f.). Fallstudien verlaufen zunächst isoliert voneinander und werden im Anschluss vergleichend betrachtet (vgl. Kapitel 7.3). 
einen orientierenden Charakter entfalten. So ist die Aufdeckung der experimentellen Varianz sowohl auf den erklärenden Variablen - den fünf AEP-Erklärungsfaktoren - als auch auf der zu erklärenden Variable - dem Policy-Mix zum barrierefreien bzw. -reduzierten Wohnraum - selbst erst ein Forschungsergebnis der Analyse. Insofern muss die Fallauswahl ohne umfassendes Wissen zur Varianz geschehen. In einer solchen Situation kann sich einerseits über Kontextvariablen genähert werden, andererseits forschungspragmatisch über persönliche Zugänge zu den Bundesländern. Beide Punkte beeinflussen in der vorliegenden Arbeit die Fallauswahl, wobei der persönliche Zugang deshalb wichtig ist, weil zahlreiche persönliche Interviews durchzuführen sind (vgl. das folgende Kapitel 5.2.2). Auf Ebene der Kontextvariablen werden geografische, sozioökonomische und demografische Kriterien betrachtet, außerdem die Wohnungsmärkte selbst. Bezüglich dieser Strukturen sollen zwei möglichst unterschiedliche Bundesländer ausgewählt werden, weil damit mutmaßlich auch diverse politische Prozesse und Policy-Outputs einhergehen. Diese Entscheidungsgrundlage wird im Folgenden skizziert (vgl. Tabelle 6). ${ }^{3}$

Tabelle 6: Kontextvariablen zu Sachsen-Anhalt und Hessen (eigene Darstellung)

\begin{tabular}{|c|c|c|c|}
\hline & $\begin{array}{l}\text { Sachsen- } \\
\text { Anhalt }\end{array}$ & Hessen & Quellen \\
\hline $\begin{array}{l}\text { Bevölkerungsdichte (Ein- } \\
\text { wohner*innen je } \mathrm{km}^{2} \text { ) }\end{array}$ & 109 & 296 & Statistisches Bundesamt 2019c \\
\hline Einwohnerzahlen & 2.208 .000 & 6.266 .000 & Statistisches Bundesamt 2019b \\
\hline $\begin{array}{l}\text { Bruttoinlandsprodukt je } \\
\text { Einwohner*in (in Euro) }\end{array}$ & 27.221 & 44.804 & Statistisches Bundesamt 2019d \\
\hline Altersquotient 2015 & 36 & 26 & Deschermeier 2017 \\
\hline $\begin{array}{l}\text { Altersquotient } 2035 \\
\text { (prognostiziert) }\end{array}$ & 66 & 33 & Deschermeier 2017 \\
\hline $\begin{array}{l}\text { Bevölkerungsentwicklung } \\
\text { bis } 2035 \text { (prognostiziert, } \\
\text { in \%) }\end{array}$ & $-10,6$ & $+2,6$ & Deschermeier 2017 \\
\hline Leerstandsquote (in \%) & 14,2 & 6,5 & $\begin{array}{l}\text { Statistisches Bundesamt 2019a, } \\
\text { aktuellste Daten für } 2014\end{array}$ \\
\hline Mietbelastungsquote (in \%) & 26,3 & 27,3 & $\begin{array}{l}\text { Statistisches Bundesamt 2019f, } \\
\text { aktuellste Daten für } 2014\end{array}$ \\
\hline $\begin{array}{l}\text { Besitz von Wohneigentum } \\
\text { (in \%) }\end{array}$ & 42 & 49 & $\begin{array}{l}\text { Statistische Ämter des Bundes und } \\
\text { der Länder 2019a, aktuellste Daten } \\
\text { für } 2011\end{array}$ \\
\hline $\begin{array}{l}\text { Anteil an Privatvermie- } \\
\text { ter*innen am Mietwohnmarkt } \\
\text { (in \%) }\end{array}$ & 37 & 66 & $\begin{array}{l}\text { BBSR 2015b, Werte übergeordnet } \\
\text { für Ost- und Westdeutschland }\end{array}$ \\
\hline
\end{tabular}


Auch wenn Sachsen-Anhalt und Hessen flächenmäßig relativ gleich groß sind (Statistisches Bundesamt 2019e), unterscheiden sich die Strukturen innerhalb der Bundesländer doch deutlich. Intern prägt Hessen eine deutliche Nord-Süd-Achse mit dem urbanen Rhein-Main-Gebiet im Süden und dem eher ländlichen Mittel- und Nordhessen (mit Ausnahme der kreisfreien Stadt Kassel). Sachsen-Anhalt wiederum kennzeichnen die drei Oberzentren Magdeburg, Halle (Saale) und Dessau-Roßlau in einem ansonsten ländlichen Raum. Ein Blick auf die Bevölkerungsdichte bestätigt die urbane Tendenz in Hessen mit einer Dichte von 296 Einwohner*innen je $\mathrm{km}^{2}$ gegenüber 109 Einwohner* innen je $\mathrm{km}^{2}$ in Sachsen-Anhalt (Statistisches Bundesamt 2019c). Auch die Einwohnerzahlen unterscheiden sich - korrelierend mit den vorherigen Merkgrößen - deutlich: Hessen liegt mit 6.266.000 Einwohner*innen deutlich vor Sachsen-Anhalt mit 2.208.000 Einwohner*innen (Statistisches Bundesamt 2019b). Die Wirtschaftskraft lässt sich anhand des Bruttoinlandsprodukts je Einwohner*in darstellen. Dieses beläuft sich für Hessen auf 44.804 Euro und ist damit überdurchschnittlich - der Bundesdurchschnitt liegt bei 39.477 Euro -, Sachsen-Anhalt bildet hingegen mit 27.221 Euro das vorletzte Bundesland (Statistisches Bundesamt 2019d).

Beim barrierefreien bzw. -reduzierten Wohnraum erscheint auch die Altersstruktur in den Ländern wichtig. Diesbezüglich hat Deschermeier (2017) mithilfe von Daten des Statistischen Bundesamtes den Altenquotient prognostiziert, welcher den Anteil der über 67-jährigen Bevölkerung beschreibt. Von 36 Prozent im Jahr 2015 steigt dieser in Sachsen-Anhalt prognostisch bis auf 66 Prozent im Jahr 2035. Die Hess"innen sind bereits im Jahr 2015 deutlich jünger (Altenquotient: 26 Prozent), und altern bis zum Jahr 2035 weniger stark (Altenquotient: 33 Prozent). Korrelierend damit wird für Sachsen-Anhalt bis zum Jahr 2035 ein Bevölkerungsschwund von -10,6 Prozent berechnet - und damit der höchste Rückgang in allen 16 Ländern -, während Hessen sogar ein Bevölkerungswachstum von +2,6 Prozent erwartet (Deschermeier 2017: 69ff.).

Die bislang aufgeführten Indikatoren haben Auswirkungen auf die Wohnungsmärkte. Ein erster Hinweis zur Entspannung oder Anspannung von Wohnungsmärkten ist die Leerstandsquote. Sie beschreibt den Anteil an leerstehenden Wohnungen und beläuft sich in Hessen auf 6,5 Prozent, in Sachsen-Anhalt mit 14,2 Prozent auf mehr als das Doppelte (aktuellste Daten für 2014, nach: Statistisches Bundesamt 2019a). Bei der Mietbelastungsquote als Anteil des Haushaltsnettoeinkommens, der für die Deckung der Bruttokaltmiete aufzubringen ist, liegen Hessen (27,3 Prozent) und SachsenAnhalt (26,3 Prozent) eng beieinander (aktuellste Daten für 2014, nach: Statistisches Bundesamt 2019f). Die Gründe für eine im Bundesvergleich eher durchschnittliche Mietbelastung unterscheiden sich dabei deutlich: Während im Südwesten höhere Einkommen auf höhere Mietpreise treffen, sind im Osten Deutschlands niedrige Mietniveaus mit einem geringeren Einkommensniveau verbunden (ablesbar aus den vorherigen Kennzahlen). Und schließlich besitzen die Hess*innen mit 49 Prozent häufiger Wohneigentum als die Sachsen-Anhalter*innen mit 42 Prozent (Statistische Ämter des Bundes und der Länder 2019a; aktuellste Angaben aus 2011). Auch die Mietermärkte selbst sind unterschiedlich organisiert: In Ostdeutschland finden sich insgesamt mehr institutionelle Anbieter"innen von Mietwohnraum, wohingegen in Westdeutschland private Kleinvermieter*innen dominieren (66\% Privatvermieter*innen in den alten Bundesländern gegenüber $37 \%$ in den neuen Bundesländern, vgl. BBSR 2015b: 15). 
Diese Strukturen mit mehr kommunalen und genossenschaftlichen Wohnungsunternehmen in den ostdeutschen Bundesländern wirken als institutionelles Erbe der DDR-Geschichte nach und beeinflussen die politischen Handlungsspielräume nach wie vor (vgl. Gerschewski 2016: 237; Conrad 2019: 204).

Zusammenfassend wird mit Sachsen-Anhalt ein ostdeutsches, ländlich geprägtes Bundesland mit einer stark alternden Bevölkerung und einer im Bundesdurchschnitt mäßigen Wirtschaftskraft ausgewählt. Mit diesen Kriterien geht ein eher entspannter Mietermarkt mit einer hohen Leerstandsquote einher. Gleichwohl wenden die zur Miete lebenden Sachsen-Anhalter*innen aufgrund ihres tendenziell geringeren Einkommensniveaus einen ähnlich hohen Anteil für ihre Bruttokaltmiete auf wie die Hess*innen. Hessen als westdeutsches Fallstudienland wiederum ist eines der wenigen deutschen Flächenländer mit einer positiven Bevölkerungsentwicklung und einer starken Wirtschaftskraft. Dabei ist es aufgrund des Rhein-Main-Gebiets im Süden und dem eher ländlichen Mittel- und Nordhessen in sich sehr heterogen. Insgesamt zeigen die hier präsentierten Merkzahlen strukturelle Unterschiede zwischen Sachsen-Anhalt und Hessen auf, die sie als diverse Fallstudienländer qualifizieren. Im Anschluss an die empirische Analyse wird überprüft, inwieweit die beiden Bundesländer, die hier als prototypische Fälle entlang ihrer Kontextvariablen ausgewählt worden sind, sich als repräsentative Fälle beweisen konnten (Blatter et al. 2007: 140ff.; Egner 2019a: 72ff.; Pickel 2016: 32; Hague/Harrop 2004: 79ff., vgl. Kapitel 8).

\subsubsection{Datenbasis: Sekundärdaten und Datenerhebung}

Nach der Begründung der Fallauswahl ist zu klären, welches Datenmaterial zur Beantwortung der Forschungsfrage genutzt werden kann. Zunächst muss jedoch auch hier ein Untersuchungszeitraum festgelegt werden. Während für die zu erklärenden Policies mit spezifischen Monaten des Jahres 2019 ein klares Datum benannt ist (vgl. Kapitel 5.1.2), lässt sich die Betrachtung der erklärenden Faktoren nicht auf einen derart engen Zeitraum begrenzen. Das liegt schon in der Natur der Sache, dass politische Entscheider*innen in unterschiedlichen Bundesländern relativ eigenständig und damit auch zeitlich versetzt handeln. Während etwa die letzte große Novellierung der Bauordnung in Sachsen-Anhalt im Jahr 2013 stattfand, liegt die 2018er Novellierung in Hessen deutlich kürzer zurück. Aus diesem Grund ist eine prozessuale Analyse notwendig, orientiert an einer Prozessanalyse zur Nachzeichnung von politischen Prozessen (Muno 2015; Starke 2015). Dabei geben die im Jahr 2019 bestehenden politischen Maßnahmen den zu untersuchenden Zeitraum vor und die dieser Maßnahmenformulierung vorangegangenen politischen Prozesse sind von Interesse.

Das genutzte Datenmaterial ist dabei in vorhandene Sekundärdaten und eigens erhobene Primärdaten zu unterscheiden. Wie im Forschungsstand (vgl. Kapitel 3) dargelegt, kann dabei nur vereinzelt auf einschlägige wissenschaftliche Literatur zurückgegriffen werden. Insofern bietet ein breites, in seiner Qualität heterogenes Feld an angewandten Studien, Leitfäden und Broschüren verschiedenster Akteure (Ministerien, Beratungsstellen, Verbände, ...) eine nicht zu vernachlässigende Datenquelle. Da sich auch dieses Datenmaterial spezifisch für Hessen und Sachsen-Anhalt schnell erschöpft, stellen politische Dokumente aus dem parlamentarischen Prozess (Plenar- und 
Ausschussprotokolle, Verbandsanhörungen, Gesetzestexte sowie Rechtsverordnungen, Anfragen) einen wertvollen Datenfundus dar. Entlang der Keywords »Barrierefreiheit« und »Wohn-« werden die Datenbänke des sachsen-anhaltischen und hessischen Landtags gesichtet, wobei sich teils auch aus den geführten Interviews neue Quellen erschließen.

Diese Datenlage wird durch leitfadengestützte Interviews mit möglichst allen am politischen Prozess beteiligten Akteuren ergänzt. Ziel ist es, dass die jeweiligen Akteure sowohl ihre Interessen und Strategien, als auch ihre Perspektive vom politischen Prozess darlegen. Schneider (2003: 113ff.) empfiehlt dabei, all jene Akteure zu berücksichtigen, die für den Untersuchungsgegenstand wichtige Leistungen bereitstellen. Dafür zeigt Tabelle 7 die ausgewählten Akteursgruppen. Neben dem akteurstheoretischen Dreiklang aus außerparlamentarischen Interessen, Parteipolitik und Ministerialverwaltung (vgl. Kapitel 4.6) stellen einerseits Gespräche mit den Landesförderinstituten, Wohnberatungsstellen sowie Wohnungsunternehmen die Perspektive der ausführenden Akteure dar, andererseits werden Akteure der Bundes- und kommunalen Ebene kontaktiert, um zu prüfen, inwieweit die politische Steuerung des barrierefreien bzw. -reduzierten Wohnraums nicht auch von diesen föderalen Ebenen aus Impulse erhält.

Tabelle 7: Übersicht über relevante Akteure als Interviewpartner"innen (eigene Darstellung)

\begin{tabular}{|l|l|l|}
\hline Kategorien & $\begin{array}{l}\text { Unter- } \\
\text { kategorien }\end{array}$ & Akteure \\
\hline $\begin{array}{l}\text { Politisch- } \\
\text { administratives } \\
\text { System }\end{array}$ & Politisch & $\begin{array}{l}\text { Fraktionen in den Landtagen (zuständige Sprecher*innen } \\
\text { für Baupolitik und/oder Sozialpolitik) }\end{array}$ \\
\cline { 2 - 3 } & Administrativ & $\begin{array}{l}\text { Ministerialverwaltungen (entsprechende Referate in den } \\
\text { Bau-/Sozialministerien) }\end{array}$ \\
\hline $\begin{array}{l}\text { Außerparlamentarische } \\
\text { Interessengruppen }\end{array}$ & $\begin{array}{l}\text { Angebotsseite von Wohnraum (inkl. Architekten-und Hand- } \\
\text { werkskammern) }\end{array}$ \\
\hline $\begin{array}{l}\text { Weitere (teils föderal übergeordnete) } \\
\text { Akteure }\end{array}$ & $\begin{array}{l}\text { Wohnungsunternehmen } \\
\text { Wohnberatungsstellen } \\
\text { Landesförderbanken } \\
\text { Kommunale Akteure (z.B. Dezernate Bauen/Soziales) }\end{array}$ \\
\hline
\end{tabular}

Die konkrete Auswahl der Interviewpartner*innen orientiert sich an Erkenntnissen aus den parlamentarischen Dokumenten: Welche Verbände beziehen in öffentlichen Anhörungen zum barrierefreien Wohnraum Stellung, welche fachpolitischen Sprecher*innen prägen die politischen Debatten? Gleichzeitig werden teilweise nach dem Schneeballprinzip weitere Akteure hinzugezogen, denen einzelne Interviewpartner" innen eine wichtige Rolle zusprechen. Für die Auswahl der Wohnungsunternehmen wird entlang der Kriterien Regionalität und Typ (genossenschaftlich, kommunal, frei finanziert) eine möglichst breite Auswahl über das Bundesland hinweg angestrebt.

Bevor die Kontaktaufnahme und die letztliche Datenbasis selbst erläutert werden, richtet sich der Blick zunächst auf die inhaltliche Ebene und damit auf den zugrundeliegenden Leitfaden. Zur Leitfadenerstellung und qualitativen Interviewführung gilt 
die einschlägige methodische Literatur als Richtschnur (vgl. Helfferich 2019; Blatter et al. 2018: 47ff.; Liebold/Trinczek 2009; Manz 2016; Porst 2019). Der Leitfaden orientiert sich an den fünf AEP-Erklärungsfaktoren (vgl. Kapitel 4) und enthält zum Teil Abweichungen für die Gruppen »politisches System« sowie »Verbände und weitere Akteure«. Dies ist insbesondere der Tatsache geschuldet, dass zusätzlich zu den Perspektiven der Interviewten auch Sach- und Fachfragen geklärt werden sollen. Für die Landesförderbanken, Wohnberatungsstellen und Wohnungsunternehmen werden stärkere Abweichungen vorgenommen, um mehr Fachwissen zu diesen spezifischen Teilbereichen generieren zu können. Die eingangs festgelegten Inhalte werden über den gesamten Erhebungsprozess größtenteils beibehalten, um eine Vergleichbarkeit der Aussagen zu gewährleisten. Dennoch finden über die Zeit einige Modifizierungen statt - insbesondere, wenn zu bestimmten Erklärungsfaktoren bereits ausreichend Material gesammelt wurde oder andere Aspekte bislang unterbelichtet geblieben sind. Insgesamt handelt es sich bei der gewählten Interviewform um eine Mischform aus Experteninterview und strukturiert-narrativem Interview (vgl. Blatter et al. 2018: 53).

Zusammengezählt werden in der Zeit vom Januar 2019 bis zum Mai 202052 Interviews durchgeführt, teils ergänzt um telefonische oder schriftliche Nachfragen. Von diesen Interviews gelten 26 Sachsen-Anhalt und 24 Hessen (vgl. Abbildung 13 und Abbildung 14). Zwei weitere Interviews sind als »übergeordnet « einzuordnen: eines mit der KfW und dem Bundesinnenministerium zum KfW-Förderprogramm sowie eines mit einem bundesweit tätigen Immobilienunternehmen.

Abbildung 13: Durchgeführte Interviews in Sachsen-Anhalt (N 26)

6

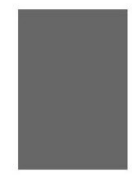

- Fraktionen

- Verbände: Angebotsseite Wohnraum

Wohnungsunternehmen

$\square$ Landesförderbanken
6

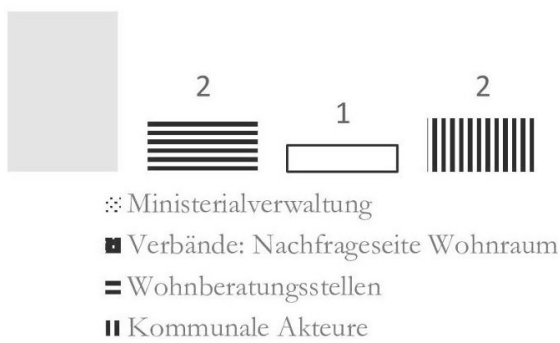

Abbildung 14: Durchgeführte Interviews in Hessen (N 24)

5

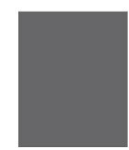

- Fraktionen

- Verbände: Angebotsseite Wohnraum

Wohnungsunternehmen

$\square$ Landesförderbanken
5

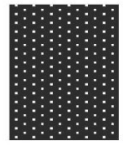

1

$\because$ Ministerialverwaltung

a Verbände: Nachfrageseite Wohntaum

= Wohnberatungsstellen

II Kommunale Akteure
4
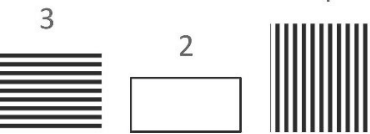

|||| 
Zur Kontaktaufnahme werden die potentiellen Interviewpartner*innen per Email angeschrieben, das Forschungsvorhaben wird skizziert und um ein circa 35- bis 45minütiges Interview zur Darstellung der eigenen Perspektive gebeten. Sofern sich die Kontaktierten nicht eigenständig zurückmelden, erfolgen zwei bis drei Wochen darauf eine oder bei Bedarf mehrere Rückfragen. Trotz dieser Anstrengungen sind bestimmte Akteure nicht zu einem Gespräch bereit, was vereinzelte »Lücken« in der Interviewübersicht erklärt. Zudem zeigt der vergleichende Blick zwei Schwerpunktverschiebungen: So werden in Sachsen-Anhalt mehr Wohnungsunternehmen interviewt als in Hessen: Der stärker institutionalisierte und eher vom Leerstand bedrohte Wohnungsmarkt in Sachsen-Anhalt ist deutlich zugänglicher, wohingegen die hessischen Wohnungsunternehmen auf ihre Landesverbände verweisen bzw. sich für den Bereich des barrierefreien/-reduzierten Wohnraums nicht sprechfähig halten (vgl. auch Kapitel 5.2.1 zu den regionalen Wohnungsmärkten). Von 15 angefragten Wohnungsunternehmen ist auch nach Nachfrage lediglich eines zu einem Gespräch bereit. In Sachsen-Anhalt liegt die Erfolgsquote mit sechs Interviews aus 13 Anfragen merklich höher. Dafür wird in Hessen wiederum die kommunale Ebene stärker berücksichtigt, da die Interviewpartner*innen in Sachsen-Anhalt die Relevanz der Kommunen betonen.

Die Interviews finden in den meisten Fällen als persönliches Gespräch bei den Akteuren vor Ort statt, in vereinzelten Fällen in einem Café oder Restaurant. Eine Ausnahme bilden die Interviews mit den Wohnungsunternehmen - aufgrund der regionalen Streuung würde das Aufsuchen vor Ort die zeitlichen und finanziellen Ressourcen des Forschungsvorhabens überreizen, sodass hier zumeist telefonische Interviews das Mittel der Wahl sind. Zudem werden alle Gespräche von März bis Mai 2020 durch die Ausbreitung der Corona-Pandemie rein telefonisch durchgeführt. In der Regel dauern die Interviews zwischen 45 und 70 Minuten. Schließlich kann ergänzend zu den Interviews am Fachgespräch »Barrierefreies Bauen in Sachsen-Anhalt - wie geht's (besser)?! « des Allgemeinen Behindertenverbandes in Sachsen-Anhalt e.V. teilgenommen werden. Bei diesem Fachgespräch im Juni 2019 sind parteipolitische Akteure und Akteure der regionalen Wohnungsmärkte anwesend, die Teilnahme erlaubt Einblicke in die politischen Forderungen eines Behindertenverbands und die entsprechenden Reaktionen. Diese Erkenntnisse werden in Form eines Beobachtungsprotokolls festgehalten und fließen ebenfalls in die empirische Analyse ein.

\subsubsection{Datenaufbereitung und -auswertung}

Parallel zur Datenerhebung findet die Datenaufbereitung in Form einer Transkription statt. So werden die Gespräche entweder mit einem Diktiergerät (persönliches Interview) oder einer Diktier-App (telefonisches Interview) mitgezeichnet und anschließend mit der Transkribier-Software $f_{4}$ transkribiert. ${ }^{4}$ Die Transkriptionsregeln passen sich

4 In einem Fall verweigern die beiden Interviewpartner*innen eine Mitzeichnung des Gesprächs, sodass hier Gesprächsnotizen - die während des Interviews verfasst werden können - als Datenmaterial dienen. 
dem Erkenntnisinteresse an. So werden die Interviews vollständig transkribiert, teils mit leichten sprachlichen Glättungen. Aktionen wie »lachen«, »überlegen« und »kurze Unterbrechungen « sind gekennzeichnet, ansonsten wird auf eine Mitschrift von Lautstärke, Gestik oder Mimik verzichtet (vgl. Geyer 2016; Kuckartz 2010: 29ff.).

Die Transkripte werden den Interviewpartner*innen zur Durchsicht und Freigabe zugesendet und zum Teil nehmen diese Änderungen, Ergänzungen oder Streichungen vor. Nach der Freigabe erfolgt die Datenauswertung als strukturierende qualitative Inhaltsanalyse: "Dabei steht der analytische Zugriff auf den manifesten Inhalt im Vordergrund, d.h. es geht vor allem darum, strukturierend und komprimierend herauszuarbeiten, was in Bezug auf die Beantwortung der Forschungsfrage im Material gesagt oder geschrieben wurde« (Blatter et al. 2018: 115, vgl. auch Kuckartz 2010: 93; Egner 2019a: 87ff.). Diese Reduktion der Datenmenge wird mithilfe der Codiersoftware MaxQDA durchgeführt. Das Codiersystem orientiert sich dabei an den fünf AEP-Erklärungsfaktoren und wird je verfeinert um die im theoretischen Kapitel gesetzten Schwerpunkte (vgl. Kapitel 4). Ergänzend dazu werden, wo notwendig, induktiv Codes aus dem Datenmaterial heraus generiert (vgl. Rädiker/Kuckartz 2019: 99ff.).

Im codierten Material liegt eine Mischung aus thematischen Codes, Faktencodes und bewertenden Codes vor. Die thematischen Codes bilden entlang der AEP-Faktoren das Grundgerüst des Codiersystems. Faktencodes zum Inhalt der einzelnen politischen Maßnahmen verhelfen dazu, die Ausprägungen der untersuchten Policies im Rahmen der typologischen Analyse besser zu verstehen. Und bewertende Codes schildern die politischen Prozesse und die daraus resultierenden Policies aus Perspektive der einzelnen Interviewpartner*innen (vgl. Kuckartz 2010: 64ff.).

\subsection{Zusammenfassung und Gütekriterien}

Kapitel 5 hat zunächst das breitere Forschungsdesign und dann das konkrete methodische Vorgehen geschildert. Mithilfe eines zweigleisigen Vorgehens aus typologischer Analyse für alle 16 Bundesländer und politikfeldanalytischen Fallstudien für SachsenAnhalt und Hessen soll sich dem Erkenntnisinteresse »Inwieweit ist barrierefreier bzw. -reduzierter Wohnraum ein Handlungsfeld für die Politik?« genähert werden.

Abschließend sei noch auf die handlungsleitenden Gütekriterien zur qualitativen Sozialforschung verwiesen. Zentral ist es dabei, die "Kontextgebundenheit « der Forschung anzuerkennen und dementsprechend eine »intersubjektive Nachvollziehbarkeit« zu gewährleisten (Blatter et al. 2007: 36). Dies erklärt die ausführliche Darlegung des methodischen Vorgehens. So kommt schon in der Datenerhebung, konkret der Interviewdurchführung, die Subjektivität der Interviewerin unausweichlich zum Tragen. Diesem Umstand ist mit der methodisch kontrollierten und reflektierten Subjektivität zu begegnen: Die Interviewsituation ist im Hinblick auf den zeitlichen und örtlichen Kontext, aber auch die Stimmung und Sympathie zwischen den Gesprächspartner*innen hin, zu reflektieren (Helfferich 2019: 683f.). Zur Datenaufbereitung wurde bereits auf die genutzten Transkriptionsregeln verwiesen. Hier ist das übergeordnete Ziel, die audio-visuelle Interviewsituation möglichst unverfälscht in eine verschriftlichte Form zu überführen. Notwendigerweise geht mit diesem Schritt aber bereits ein Informati- 